\title{
Liver Abscess Turned Metastatic Infection in an Otherwise Healthy Patient: A Case Report
}

\author{
Samik Shah, MD, Ritu Nahar, MD, and Neha Etherington, MD
}

\section{INTRODUCTION}

The most common type of visceral abscesses are liver abscesses, which have a mortality rate of at least 2.5 percent. ${ }^{1,2}$ Most liver abscesses are polymicrobial, containing both facultative and anaerobic enteric pathogens, and develop secondary to another infection such as peritonitis and cholangitis, or from hematogenous spread. ${ }^{2-4}$ Liver abscesses are sometimes associated with systemic diseases such as colorectal cancer and diabetes mellitus.,6 Rarely, as in our case presentation, primary liver abscesses occur spontaneously in patients with no identifiable precipitating or predisposing conditions.

\section{CASE PRESENTATION}

A 54-year-old Chinese-American woman with no significant past medical history presented to the emergency room with two weeks of subjective fevers and chills that worsened two days prior to presentation. She denied shortness of breath, chest pain, abdominal pain, nausea, vomiting, diarrhea, and dysuria. She had no prior surgeries and took no medications. She did not use tobacco, alcohol, or illicit drugs and she worked in a restaurant. She immigrated to the United States from China in the 1990s, and recently traveled to China six months prior to admission.

In the emergency room, she was febrile to $101.6^{\circ} \mathrm{F}$, tachycardic to 128 beats/minute, and hypotensive to $85 / 53 \mathrm{mmHg}$. Physical exam revealed cervical lymphadenopathy. She was not jaundiced, had no abdominal tenderness or distension, and had a non-focal neurologic exam. Laboratory studies were notable for an elevated leukocyte count $15.2 \mathrm{~B} / \mathrm{L}$, aspartate aminotransferase 142 $\mathrm{IU} / \mathrm{L}$, alanine aminotransferase $189 \mathrm{IU} / \mathrm{L}$, alkaline phosphatase $295 \mathrm{IU} / \mathrm{L}$, and total bilirubin $1.3 \mathrm{mg} / \mathrm{dL}$. Abdominal ultrasound showed nonspecific gallbladder wall thickening and sludge without biliary ductal dilatation or pericholecystic fluid. Blood cultures were drawn. She was treated with fluid resuscitation and antibiotics (vancomycin, piperacillin-tazobactam, and one dose of tobramycin) for presumed sepsis.

Within 24 hours, blood cultures became positive with gram-negative bacilli, so vancomycin was stopped. Soon after, the patient developed nausea, vomiting, and a brief new oxygen requirement of $2 \mathrm{~L} / \mathrm{min}$ via nasal cannula. Chest $x$-ray revealed a new (since admission) small opacity in the left lung base. Respiratory pathogen panel, rapid influenza and respiratory syncytial virus

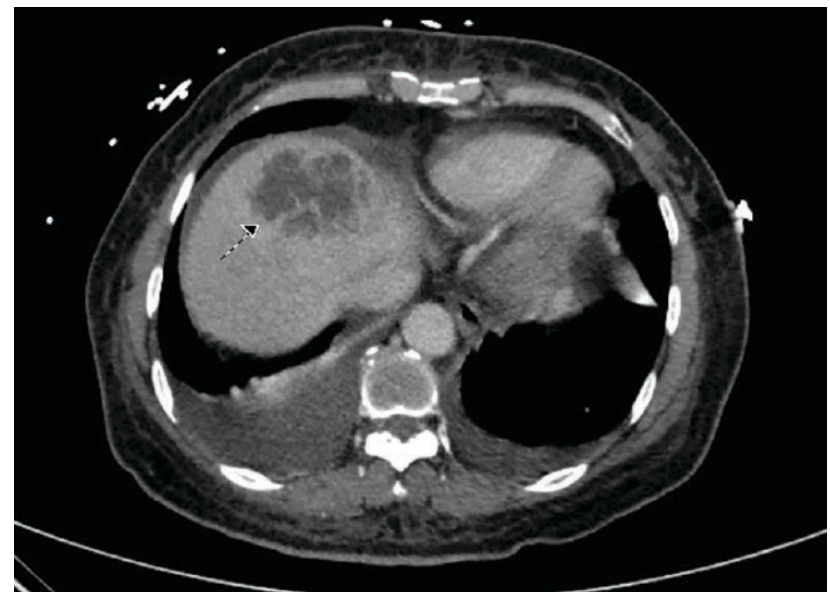

Figure 1. Computed tomography scan of the abdomen with intravenous contrast showing the abscess at the dome of the liver (arrow).

nasal swab, and urine Streptococcal and Legionella antigens were all negative.

On day three of admission, the initial blood cultures speciated as Klebsiella pneumoniae. On day four, she developed vertigo with left ear hearing loss, tinnitus, nausea, and vomiting. Dix-Hallpike maneuver was positive, but the rest of the neurologic examination was non-focal. Repeat blood cultures were persistently positive for the same organism.

\section{DIFFERENTIAL DIAGNOSIS}

The patient presented with sepsis from $K$. pneumoniae bacteremia that was complicated by hypoxia. Even with the hypoxia considered, K. pneumoniae is not a common cause of community acquired pneumonia in an otherwise healthy patient, and the patient had sustained bacteremia despite treatment with appropriate antibiotics. Her hypoxia was thus attributed to aspiration due to her emesis. Although a primary source for Klebsiella bacteremia may not be identified in up to 30-47 percent of patients, this is more common in nosocomial rather than community acquired infections.? Community acquired bacteremia from primary liver abscesses is well-documented in the East Asian population. ${ }^{8.9}$ Further, primary Klebsiella liver abscesses can rarely be complicated by metastatic brain abscesses. ${ }^{10}$ In the setting of our patient's sustained bacteremia with new onset vertigo, she underwent imaging of the brain, abdomen, and pelvis to rule out the presence of abscesses. 


\section{OUTCOME AND FOLLOW-UP}

Computed tomography (CT) of the abdomen and pelvis showed a $5.3 \times 4.6 \mathrm{~cm}$ abscess at the dome of the liver (Figure 1). CT of the head showed multiple subcortical ring-enhancing lesions (largest measuring $12 \mathrm{~mm}$ ) that were concerning for cerebral abscesses. Magnetic resonance imaging (MRI) of the brain similarly showed multiple cerebral abscesses. The cerebral abscesses were determined to be too small for neurosurgical intervention. MRI of the whole spine was unremarkable. Her antibiotics were narrowed from piperacillin-tazobactam to ceftriaxone for better central nervous system penetration. On day five of admission, she underwent liver abscess drain placement with fluid culture positive for $K$. pneumoniae. The liver abscess drain was removed after four days because of decreased output.

After the drain placement, blood cultures cleared and remained negative. Repeat brain MRI performed six days after the initial scan showed that some lesions increased and others decreased in growth, and that there was a new punctate foci of enhancement. Neurological exam remained non-focal. On day 13, she was discharged home to complete a six-week course of intravenous ceftriaxone. Post-discharge imaging shows resolution of the liver abscesses. Unfortunately, she had persistent tinnitus and difficulty hearing on the left side, so she sees an audiologist.

\section{DISCUSSION}

This patient's K. pneumoniae bacteremia and multiple brain abscesses were likely complications of her $5.3 \times 4.6$ $\mathrm{cm}$ liver abscess since blood cultures became negative after the abscess was drained. The patient had no predisposing conditions including hepatobiliary or colorectal disease, recent intra-abdominal surgery, trauma, or immunocompromising factors. Ko et al. determined the liver to be the primary source in $18 \%$ of Taiwanese patients with community-acquired Klebsiella bacteremia, as opposed to just $2 \%$ in other countries, including the United States and Australia. ${ }^{11}$ Among patients with $K$. pneumoniae liver abscesses, Fang et al. found that $23 \%$ had septic ocular or central nervous system metastasis, while Cheng et al. found that $12 \%$ of patients had some sort of metastatic lesion, also including endophthalmitis, pulmonary abscesses, and central nervous system abscesses. ${ }^{8,12}$

Diabetes mellitus, fatty liver disease, and certain virulence factors found on the specific Klebsiella organisms such as a bacterial capsular serotype resistant to phagocytosis, are considered to be risk factors for the pathogenesis of this. ${ }^{8.9}$

In a study of 79 patients with liver abscesses from any organism, the most common presenting symptoms were found to be fever, chills, right upper quadrant abdominal pain, nausea, and vomiting. The most common laboratory abnormalities involved the initial serum albumin (mean $3.1 \mathrm{~g} / \mathrm{dL}, 70 \%$ of patients had an abnormal value), leukocyte count (15.4 B/L, 68\%), alkaline phosphatase (206.8 IU/L, 67\%), and alanine aminotransferase (93.4 IU/L, 54\%). ${ }^{2}$ Treatment of the liver abscesses involves drainage of the abscess to obtain source control. Antibiotics should be tailored to the sensitivities of the Klebsiella species, with a duration of at least a four to six weeks. Follow-up CT imaging is recommended to ensure resolution of the abscesses.

\section{KEY POINTS}

This case highlights the importance of a broad differential diagnosis when evaluating a patient with an unknown source of bacteremia. A Klebsiella liver abscess should be considered in East Asian patients without an identified source of bacteremia. Early detection to prevent central nervous system metastasis can prevent further devastating complications. The identification of our patient's liver abscess allowed for source-directed therapy with immediate drainage and subsequent resolution of the patient's bacteremia.

\section{REFERENCES}

1. Altemeier WA, Culbertson WR, Fullen WD, et al. Intra-abdominal abscesses. Am J Surg. 1973;125(1):70-9

2. Rahimian J, Wilson T, Oram V, et al. Pyogenic liver abscess: recent trends in etiology and mortality. Clin Infect Dis. 2004; 39(11):1654-9.

3. Huang CJ, Pitt HA, Lipsett PA, et al. Pyogenic hepatic abscess. Changing trends over 42 years. Ann Surg. 1996; 223(5):600-7.

4. Leggieri N, Marques-Vidal P, Cerwenka H, et al. Migrated foreign body liver abscess: illustrative case report, systematic review, and proposed diagnostic algorithm. Medicine (Baltimore). 2010; 89(2):85-95.

5. Kao WY, Hwang CY, Chang YT, et al. Cancer risk in patients with pyogenic liver abscess: a nationwide cohort study. Aliment Pharmacol Ther. 2012;36(5):467-76.

6. Thomsen RW, Jepsen P, Sørensen HT. Diabetes mellitus and pyogenic liver abscess: risk and prognosis. Clin Infect Dis. 2007;1;44(9):1194-201.

7. Tsay RW, Siu LK, Fung CP, et al. Characteristics of bacteremia between community-acquired and nosocomial Klebsiella pneumoniae infection: risk factor for mortality and the impact of capsular serotypes as a herald for communityacquired infection. Arch Intern Med. 2002;162:1021.

8. Fang CT, Lai SY, Yi WC, et al. Klebsiella pneumoniae genotype K1: an emerging pathogen that causes septic ocular or central nervous system complications from pyogenic liver abscess. Clin Infect Dis. 2007:45:284.

9. Wang JH, Liu YC, Lee SS, et al. Primary liver abscess due to Klebsiella pneumoniae in Taiwan. Clin Infect Dis. 1998:26(6):1434

10. Ni YH, Yeh KM, Peng MY, et al. Community-acquired brain abscess in Taiwan: etiology and probable source of infection. J Microbiol Immunol Infect. 2004; $37: 231$.

11. Ko WC, Paterson DL, Sagnimeni AJ, et al. Community-acquired Klebsiella pneumoniae bacteremia: global differences in clinical patterns. Emerg Infect Dis. 2002:8(2):160-6

12. Cheng DL, Liu YC, Yen MY, et al. Septic metastatic lesions of pyogenic liver abscess. Their association with Klebsiella pneumoniae bacteremia in diabetic patients. Arch Intern Med. 1991;151(8):1557-9. 